\title{
Information zur Methadonabgabe
}

Tarifdienst der FMH
Im Herbst 2005 hat Sie die FMH betreffend einer Anpassung der medizinischen Interpretation der Position 00.0150 in der künftigen TARMED-Fassung 1.03 informiert, die nun am 1. April 2006 in Kraft treten wird. Diese Position wird ab diesem Datum für die Verrechnung der Methadonabgabe durch nichtärztliches Praxispersonal nicht länger verwendet werden dürfen.

Damals empfahl die FMH den kantonalen Ärztegesellschaften und allen betroffenen Einrichtungen oder Institutionen, direkt mit den Versicherern Lösungen zu suchen. Diese Verhandlungen haben bis jetzt jedoch zu keinem Ergebnis geführt.

Um einer tarifarischen Lücke vorzubeugen, hat die FMH mit santésuisse einen Übergangsvertrag vereinbart. Es wird demzufolge weiterhin möglich sein, ab dem 1. April 2006 die Methadonabgabe in der Arztpraxis durch nichtärztliches Personal zu verrechnen. Aus juristischen und technischen Gründen war es jedoch nicht möglich, einfach die bisherige Fakturierungsweise beizubehalten.

Folgende Lösung wurde gefunden: Eine Pauschale von Fr. 40.- pro Woche, welche spezifisch die Vorbereitung der Dosen und die Methadonabgabe durch nichtärztliches Personal betrifft. Der Code für die Leistung (auf der Rechnung) ist 999, die Bezeichnung «PMETH 00.0150». Die betroffenen Ärztinnen und Ärzte müssen sich direkt bei santésuisse anmelden.

Es ist nun Sache der FMH, mit santésuisse eine langfristige Pauschallösung auszuhandeln, die mit dem Kapitel 8, Anhang 1, der KLV übereinstimmt. Ein erster Vertragsentwurf wurde bereits mehreren direkt betroffenen Kollegen, Kantonen und Organisationen zur Stellungnahme vorgelegt. santésuisse hat ihrerseits signalisiert, dass sie an einer Lösung auf Vertragsbasis interessiert sei.

\section{Information sur la remise de méthadone}

Service tarifaire FMH

La FMH vous avait informé, en automne 2005, d'une modification quant à l'interprétation de la position 00.0150 dans la version TARMED 1.03, dont l'entrée en vigueur est fixée maintenant au $1^{\text {er }}$ avril 2006. On ne pourra plus dès lors facturer de la même manière la remise de la méthadone aux patients par le personnel non médical du cabinet.

La FMH avait à l'époque proposé aux sociétés cantonales et à toutes les structures et institutions concernées de rechercher des solutions directes avec les assureurs. Ces négociations n'ont pas abouti à ce jour.

Pour éviter un vide tarifaire, la FMH a négocié un accord transitoire avec santésuisse. Il sera ainsi possible, à partir du $1^{\text {er }}$ avril 2006, de continuer à facturer la remise de la méthadone en cabinet par du personnel non médical, mais pour des raisons légales et techniques, il n'était pas possible de simplement garder le système de facturation actuel.

La solution retenue est celle d'un forfait de Fr. 40.- par semaine, qui concerne spécifiquement la préparation des doses et la remise de méthadone par du personnel non médical; le code de la prestation (sur la facture) sera 999, et le libellé «PMETH 00.0150». Les médecins concernés devront s'inscrire directement auprès de santésuisse.

Il appartiendra maintenant à la FMH de négocier avec santésuisse une solution forfaitaire à long terme, en accord avec le chapitre 8 de l'annexe 1 de l'OPAS. Dans ce but, une première ébauche de contrat a déjà été soumise pour prise de position à plusieurs collègues, cantons et organisations directement concernés. santésuisse a par ailleurs signalé sa volonté d'aboutir à une solution négociée. 